\title{
Ras SSDNA aptamer inhibits vascular smooth muscle cell proliferation and migration through MAPK and PI3K pathways
}

\author{
XIAOPING HU, ZHIWEI WANG, HONGBING WU, WANLI JIANG and RUI HU \\ Department of Cardiovascular Surgery, Wuhan University Renmin Hospital, Wuhan, Hubei 430060, P.R. China
}

Received June 11, 2014; Accepted February 25, 2015

DOI: $10.3892 /$ ijmm.2015.2139

\begin{abstract}
Proliferation and migration of vascular smooth muscle cells (VSMCs) mediated by Ras proteins are crucial in restenosis following percutaneous coronary intervention (PCI) and coronary artery bypass graft (CABG). In this study, a novel, single-stranded DNA (ssDNA) aptamer designated as Ras-a1 with high affinity and specificity to human Ras protein was isolated using systematic evolution of ligands by exponential enrichment. Ras-a1 was delivered into VSMCs by electroporation using one square waveform of $200 \mathrm{~V}$ for $20 \mathrm{msec}$. Proliferation of VSMCs was determined using a cell counting kit-8 assay, which revealed the maximal inhibitory rate $(40 \%)$ was obtained at $24 \mathrm{~h}$ after Ras-a1 transfection. The migration of VSMCs, determined using a Transwell assay, was significantly inhibited in Ras-al cells in a time-dependent manner. To investigate the potential mechanisms of transfected Ras-al on the migration and proliferation of VSMCs, the phosphorylation of MEK1/2, ERK1/2, and Akt was determined using western blot analysis, which showed that a marked downregulation was observed in the phosphorylation of MEK1/2, ERK1/2, and Akt following the delivery of Ras-a1. This result demonstrated that Ras-a1 inhibits the proliferation and migration of VSMCs by inhibiting the phosphorylation of Ras and interrupting signal transduction in the Ras-MEK1/2-ERK1/2 and phosphoinositide-3 kinase/Akt pathways. The novel Ras protein-targeted ssDNA aptamer selected may be applicable for the prevention of restenosis after PCI and CABG.
\end{abstract}

\section{Introduction}

Restenosis remains a challenge to percutaneous coronary intervention (PCI) and coronary artery bypass graft (CABG) for patients with coronary artery diseases (CHD) $(1,2)$. The proliferation and migration of vascular smooth muscle cells

Correspondence to: DrZhiwei Wang, Department of Cardiovascular Surgery, Wuhan University Renmin Hospital, 238 Jiefang Road, Wuhan, Hubei 430060, P.R. China

E-mail:wangzhiwp@163.com

Key words: Ras protein, aptamer, vascular smooth muscle cells, restenosis, PI3K/Akt pathway
(VSMCs) has been considered the major cause for underlying restenosis after PCI or CABG $(3,4)$. To the best of our knowledge, the activation of mitogen-activated protein kinase (MAPK) and phosphoinositide-3 kinase (PI3K) signaling pathways mediated by Ras protein play a pivotal role in the proliferation of VSMCs in the presence of vascular injury $(5,6)$. Consequently, we hypothesized that blockage of Ras protein activation may be a target for therapeutic strategy for vascular restenosis after PCI and CABG.

Using the systematic evolution of ligands by exponential enrichment (SELEX) technique, several aptamers with high affinity and specificity for target proteins were previously selected from a library of randomized sequences in vitro, including single-stranded DNA (ssDNA) or RNA oligonucleotides (7-9). For instance, Bell et al (10) developed an oligonucleotide designated as NX1838 (2'-fluoropyrimidine, RNA-based oligonucleotide/40-kDa-PEG) that inhibited vascular endothelial growth factor 165 (VEGF165)-mediated cell response in vitro by directly targeting the VEGF165 factor. In addition, an oligonucleotide inhibiting multiple interferon- $\gamma$ signaling transduction was isolated, which could block the binding of interferon- $\gamma$ to its receptor (11). However, few studies have been carried out to develop a specific aptamer binding directly to the Ras protein.

In this study, a Ras protein-targeted ssDNA aptamer designated as Ras-a1 was identified, and its potential function in the proliferation and migration of human saphenous VSMCs was investigated. Our study indicated that Ras-a1 inhibited the proliferation and migration of VSMCs, and suppressed the phosphorylation of proteins involved in the MEK, ERK1/2 and Akt signaling pathways.

\section{Materials and methods}

Materials. Dulbecco's modified Eagle's medium with high glucose (DMEM-HG), Opti-MEM I medium, Dulbecco's phosphate-buffered saline (PBS), glutamine, trypsin-EDTA, and penicillin/streptomycin were purchased from Invitrogen (Carlsbad, CA, USA). Fetal bovine serum (FBS) was purchased from Thermo Scientific (Logan, UT, USA). Cell Counting kit-8 (CCK-8) was purchased from Dojindo Laboratories (Kumamoto, Japan). The Transwell assay with $8-\mu \mathrm{m}$ pore polycarbonate membrane was purchased from Corning Inc. (Corning, NY, USA). Rabbit polyclonal anti-phospho-MEK1/2, 
rabbit polyclonal anti-phospho-ERK1/2, rabbit polyclonal anti-phospho-Akt, mouse polyclonal anti-GAPDH, mouse polyclonal anti-Ras, mouse polyclonal anti-MEK1/2, mouse polyclonal anti-ERK1/2, and mouse polyclonal anti-Akt antibodies were purchased from Cell Signaling Technology (Beverly, MA, USA).

Cell culture. VSMCs were cultured in DMEM-HG medium supplemented with $10 \% \mathrm{FBS}, 100 \mathrm{U} / \mathrm{ml}$ penicillin and $100 \mu \mathrm{g} / \mathrm{ml}$ streptomycin. Cultures were maintained at $37^{\circ} \mathrm{C}$ in a humidified $95 \%$ air and $5 \% \mathrm{CO}_{2}$ atmosphere. The primary cells were obtained from three patients with stenosis. All experiments were performed in cell passages of 4-8. Written informed consent was obtained from each patient. The study protocols were approved by the Ethics Committee of Renmin Hospital of Wuhan University.

Random DNA library and primers. The DNA library was produced using the sequence, 5'-GGGAGCTCAGAATAAA CGCTCAA- ${ }_{35}{ }^{-}$TTCGACATGAGGCCCGGATC-3', where central $\mathrm{N}_{35}$ represented the random incorporation of $A, G, C$ and $\mathrm{T}$ at each position. The initial dsDNA random library was generated by PCR amplification using forward, 5'-GGGAGCTCA GAATAAACGCTCAA-3' and the biotin-labelled reverse 5'-GATCCGGGCCTCATGTCGAA-3' primers.

In vitro selection of Ras ssDNA aptamers. For the selection of Ras ssDNA aptamers in vitro, Ras protein (100 pmol) and sterile $\mathrm{dsH}_{2} \mathrm{O}(100 \mu \mathrm{l})$ were incubated with $100 \mu \mathrm{l}$ coating buffer $\left(0.05 \mathrm{~mol} / 1 \mathrm{NaHCO}_{3}\right.$, PH 9.6) in 96-well plates for $18 \mathrm{~h}$ at $4^{\circ} \mathrm{C}$. After washing with PBS, the wells were blocked with $200 \mu 13 \%$ bovine serum albumin (BSA) for $45 \mathrm{~min}$ at $37^{\circ} \mathrm{C}$. Subsequently, $100 \mu \mathrm{l}$ random ssDNA pools were incubated with $100 \mu \mathrm{l}$ binding buffer (20 mM Hepes $\mathrm{pH} 7.35,120 \mathrm{mM}$ $\mathrm{NaCl}, 5 \mathrm{mM} \mathrm{KCl}, 1 \mathrm{mM} \mathrm{MgCl}_{2}$, and $1 \mathrm{mM} \mathrm{CaCl}_{2}$ ) for $45 \mathrm{~min}$ at $37^{\circ} \mathrm{C}$. The supernatants were then incubated for another $45 \mathrm{~min}$ at $37^{\circ} \mathrm{C}$. Following the removal of the unbound sequences, well-retained ssDNA was eluted by adding the elution buffer (20 mM Tris- $\mathrm{HCl}, 4 \mathrm{~mol} / \mathrm{l}$ guandine thiocyanate, and $1 \mathrm{mM}$ DTT, pH 8.3) and incubated at $80^{\circ} \mathrm{C}$ for $8 \mathrm{~min}$. Ethanol was then added to precipitate the ssDNA products.

The isolated ssDNA was amplified by PCR reactions performed in a total volume of $20 \mu 1$ of PCR mixtures, containing $10 \mu \mathrm{l}$ of $2 \mathrm{X}$ PCR buffer, $0.4 \mathrm{mM}$ dNTPs, $10 \mathrm{pmol}$ forward primers, $10 \mathrm{pmol}$ reverse biotinylated primers, $1 \mathrm{pmol}$ template, and 0.5 units Taq DNA polymerase. The PCR conditions were: denaturation at $94^{\circ} \mathrm{C}$ for $3 \mathrm{~min}$, followed by 24 cycles of denaturation at $94^{\circ} \mathrm{C}$ for $40 \mathrm{sec}$, annealing at $60^{\circ} \mathrm{C}$ for $30 \mathrm{sec}$, and extension at $72^{\circ} \mathrm{C}$ for $30 \mathrm{sec}$. PCR products were electrophoresed on a $3 \%$ agarose gel and then purified by a UNIQ-10 DNA Extraction kit (Sangon, Shanghai, China) according to the manufacturer's instructions.

The ssDNA pool was obtained by separating the dsDNA PCR product with streptavidin-coated magnetic beads (M-280 Dynabeads; Invitrogen, Karlsruhe, Germany). Briefly, $50 \mu \mathrm{g}$ dsDNA PCR products were incubated with $10 \mathrm{mg}$ beads in a tube on a rotator for $30 \mathrm{~min}$. Subsequently, the tube was placed on a magnet for $3 \mathrm{~min}$ to obtain the biotinylated dsDNA. The coated beads were washed three times in binding and washing buffer (10 mM Tris-HCl, 1 mM EDTA, $2 \mathrm{M} \mathrm{NaCl}$ ). Alkaline denaturation was performed with $0.15 \mathrm{M} \mathrm{NaOH}$ for 2 min. Subsequently, the supernatant was collected, and the selected ssDNA aptamer for the following SELEX round was successfully obtained. The repeated selection and amplification were performed until no significant increase was evident in the binding rates and affinities.

Binding affinity assays between the aptamer pools and Ras protein. The ELISA assay was used to examine the direct binding of aptamer to Ras protein. Ras protein was used to coat Nunc 96 -well plates and incubated overnight at $4^{\circ} \mathrm{C}$. After washing with PBS, the wells were blocked with 3\% BSA for $45 \mathrm{~min}$ at $37^{\circ} \mathrm{C}$. The obtained biotinylated aptamers (up to $100 \mathrm{nM}$ ) were allowed to bind with Ras protein for $45 \mathrm{~min}$ at $37^{\circ} \mathrm{C}$. The plates was washed again, and streptavidin-HRP conjugate was applied as a secondary detection reagent. The plate was washed, and tetramethyl-benzidine substrate was added. For color development, a wavelength of $450 \mathrm{~nm}$ was used for the light absorbance measured by using an ELISA reader (Lumitron Ltd., Petach-Tikva, Israel). The dissociation constant $(\mathrm{Kd})$ was calculated according to the formula $\mathrm{Y}=\mathrm{B}_{\max } \mathrm{x} \mathrm{X} /(\mathrm{Kd}+\mathrm{X})$, where $\mathrm{X}$ was the concentration, $\mathrm{Y}$ the mean optical density, and $\mathrm{B}_{\max }$ the maximum optical density.

Cloning, sequencing and structure analysis of selected aptamers. The selected aptamers from the 11th round of SELEX were purified and linked with pMD18-T vector, and then transformed into Escherichia coli DH5 $\alpha$ strains. Plasmid DNA was isolated from individual clones, purified and analyzed by sequencing. A total of 45 individual aptamers were obtained. The primary and secondary structures of individual aptamers were analyzed based on their sequences using the DNAMAN 5.29 software (Lynnon Corp., Quebec, QC, Canada).

Transfection of Ras-al. Prior to transfection, the cells were washed twice with Opti-MEM I medium and resuspended in Opti-MEM I medium until a final concentration of $2.0 \times 10^{6}$ cells $/ \mathrm{ml}$. After the addition of Ras-al $(20 \mu \mathrm{g} / \mathrm{ml})$, the cells were cooled on ice for $10 \mathrm{~min}$. Subsequently, $400 \mu \mathrm{l}$ cell suspension was transferred to a 0.4-cm-gap cuvette (BTX, Holliston, MA, USA). Subsequently, electroporation was performed using one pulse of $200 \mathrm{~V}$ electric field and $20 \mathrm{msec}$ pulse length using ECM 830 Electro Square Porator (BTX). The cells treated using only electroporation were set as the control. Following electroporation, the cells were incubated at $37^{\circ} \mathrm{C}$ in a humidified $95 \%$ air and $5 \% \mathrm{CO}_{2}$ atmosphere for $30 \mathrm{~min}$. For the assessment of cell viability, $50 \mu \mathrm{l}$ cell suspension obtained from each cuvette was transferred to a 96-well plate with $1 \times 10^{4}$ cells per well and cultured for $12 \mathrm{~h}$. To assess the efficiency of transfection, $200 \mu \mathrm{l}$ cell suspension from each cuvette was plated in a 24 -well plate containing $1 \mathrm{ml}$ DMEM-HG medium supplemented with $10 \%$ FBS, followed by incubation for $6 \mathrm{~h}$.

Cell proliferation assay. The proliferation of VSMCs was determined using a CCK-8 kit according to the manufacturer's instructions. Briefly, the cells were collected $30 \mathrm{~min}$ after electroporation. The cells were washed using PBS, and resuspended in DMEM-HG medium supplemented with $10 \%$ FBS. 
A

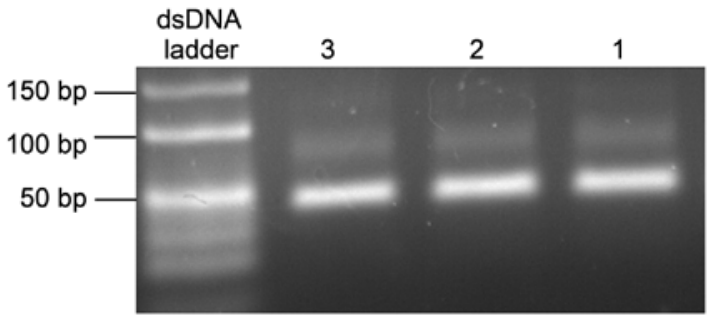

B Minimum free energy of the structure $-9.77 \mathrm{kcal} / \mathrm{mol}$

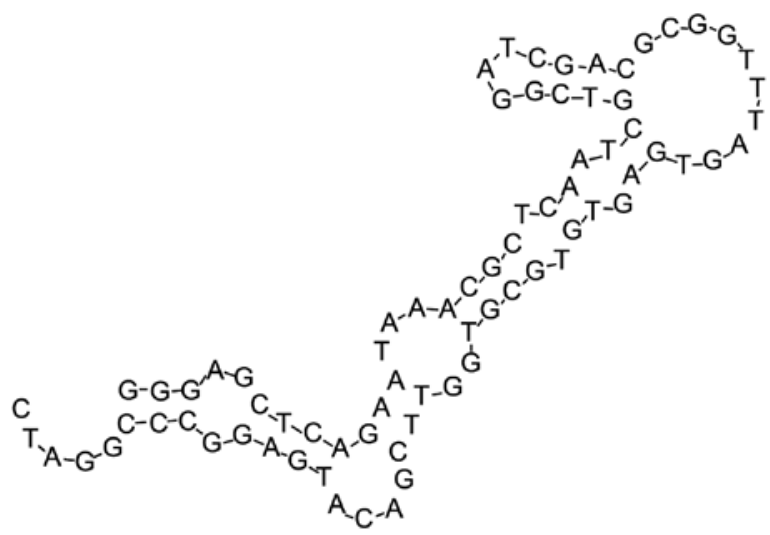

Figure 1. The amplification results were electrophoresed on 3\% agarose gel. (A) Lanes 1-3 were the products of the 1st, 5th, and 11th round of selection, respectively. (B) The secondary structure of Ras-a1 was predicted and revealed small stem-loops in Ras-a1.

Subsequently, $100 \mu 1$ cell suspension was added into each well of the 96 -well plate until a concentration of $1 \times 10^{5}$ cells $/ \mathrm{ml}$. The cells were then collected at $12,24,36$ and $48 \mathrm{~h}$, respectively. At each time-point, $10 \mu$ l CCK-8 reagent was added to each well and incubated for another $2 \mathrm{~h}$ prior to measuring the optical density (OD) in each well at $450 \mathrm{~nm}$ with a VICTOR3 Multilabel Counter model 1420 (Elmer-Perkin, Boston, MA, USA). The inhibitory rates were calculated using the formula: Inhibitory rate $(\%)=1-\mathrm{OD}$ (Ras-a1 cells)/OD (control cells) x100.

Cell migration assay. The migration of VSMCs was measured using Transwell chambers with $8.0-\mu \mathrm{m}$ pore polycarbonate membranes as previously described (12). Briefly, the cells were harvested $30 \mathrm{~min}$ after electroporation. After washing with PBS, the cells were resuspended in serum-free DMEM supplemented with $0.2 \%$ BSA. Cell suspension $(100 \mu \mathrm{l})$ was then transferred into the upper chamber at a concentration of $1 \times 10^{5}$ cells $/ \mathrm{ml}$, with the lower chambers filled with $600 \mu \mathrm{l}$ DMEM-HG medium supplemented with $10 \%$ FBS. The chambers were incubated in a cell culture incubator for 6, 12, 18 and $24 \mathrm{~h}$ at $37^{\circ} \mathrm{C}$, respectively. At each time-point, the cells on the upper surface of the polycarbonate membrane were gently removed, while the cells on the reverse side of the membrane were fixed with $4 \%$ paraformaldehyde for $15 \mathrm{~min}$ and stained with DAPI for $10 \mathrm{~min}$. Cell migration was quantified by counting the number of cells on the reverse side of the polycarbonate membrane under an IX51 converted fluorescence microscope (Olympus Corporation, Tokyo, Japan). Five fields were randomly selected in each membrane for the cell count. The experiments were performed at least in triplicate.
Western blot analysis. MEK1/2, ERK1/2 and Akt protein expression in VSMCs was determined using western blot analysis as previously described (13). Briefly, the cultured VSMCs were lysed at $4^{\circ} \mathrm{C}$ in lysis buffer containing $100 \mathrm{mmol} / \mathrm{l}$ Tris- $\mathrm{HCl} \mathrm{pH} 7.4,150 \mathrm{mmol} / \mathrm{l} \mathrm{NaCl}, 1 \% \mathrm{NP}-40$, $1 \%$ sodium deoxycholate, $5 \mathrm{mmol} / 1$ EDTA, $10 \mu \mathrm{g} / \mathrm{ml}$ leupeptin, $10 \mu \mathrm{g} / \mathrm{ml}$ aprotinin, $10 \mu \mathrm{g} / \mathrm{ml}$ pepstain, $1 \mathrm{mmol} / 1$ PMSF, $10 \mathrm{mmol} / 1 \quad \beta$-glycerophosphate, $1 \mathrm{mmol} / 1 \mathrm{Na}_{3} \mathrm{VO}_{4}$ and $10 \mathrm{mmol} / 1 \mathrm{NaF}$. Subsequently, $50 \mu \mathrm{g}$ proteins were separated via $10 \%$ SDS-PAGE and transferred onto nitrocellulose membranes (Pall Corporation, Port Washington, NY, USA). The membranes were then blocked in Tris-buffered saline (TBS) containing 5\% non-fat milk for $2 \mathrm{~h}$. The mixture was then incubated in the primary antibody including rabbit polyclonal anti-phospho-MEK1/2 (1:1,000), anti-phospho-ERK1/2 $(1: 2,000)$, anti-phospho-Akt $(1: 1,000)$, mouse polyclonal anti-GAPDH (1:500), anti-Ras $(1: 1,000)$, anti-MEK1/2 $(1: 1,000)$, anti-ERK1/2 $(1: 2,000)$, or anti-Akt $(1: 1,000)$ antibody in TBST containing $1 \%$ BSA overnight at $4^{\circ} \mathrm{C}$. The membranes were then washed and incubated in IRDye $800 \mathrm{CW}$ goat anti-rabbit/mouse IgG secondary antibodies $(1: 10,000)$ for detection. The same membrane was probed for GAPDH for the loading control. Intensity of the protein band was determined using an Odyssey Infrared Imaging System (LI-COR Biotechnology, Lincoln, NE, USA).

Statistical analysis. Data are expressed as the means \pm standard deviation (SD). ANOVA or the unpaired Student's t-test was used to determine the inter-group difference. $\mathrm{P}<0.05$ was considered statistically significant.

\section{Results}

Isolation of high-affinity Ras protein aptamers. In this study, Ras protein was used as a selection target, while random ssDNA library was used as screening ligands. Sterile $\mathrm{ds}_{2} \mathrm{O}$ was used for the counter selection. After 11 rounds of selection, different pools of aptamers were obtained using SELEX. The aptamers of each pool and the PCR products were identified using electrophoresis on a $3 \%$ agarose gel. The length of the dsDNA ranged $40-50$ bp (Fig. 1A).

As revealed by the ELISA assay, the binding affinities increased gradually from 51.5 to $18.3 \mathrm{nM}(\mathrm{Kd})$. The highest binding affinity was noted in the 11th pool with a Kd of $18.3 \mathrm{nM}$. No further improvement was observed in the binding affinities obtained after 11 rounds of selection. Subsequently, the products selected through the 11th pool were linked to the pMD18-T vector, based on which the individual clones of single aptamers were selected and sequenced. The consensus regions within the individual aptamers are shown in Table I. As shown in Tables II and III, a total of 45 individual aptamers were obtained. For the binding affinities between individual aptamers and Ras, Ras-a1 showed the highest affinity with a $\mathrm{Kd}$ of $15.3 \mathrm{nM}$. The sequence of binding affinities between the aptamer pools was as follows: Ras-a1 $>11$ th pool $>10$ th pool $>9$ th pool $>8$ th pool $>6$ th pool $>4$ th pool $>$ Ras-a27 $>1$ st pool (Table II). DNAMAN 5.29 software (Lynnon Corp.) was used to predict the secondary structure of Ras-a1, which showed that the binding sites to the Ras protein were localized at the terminal loop of a stem-loop structure (Fig. 1B). 


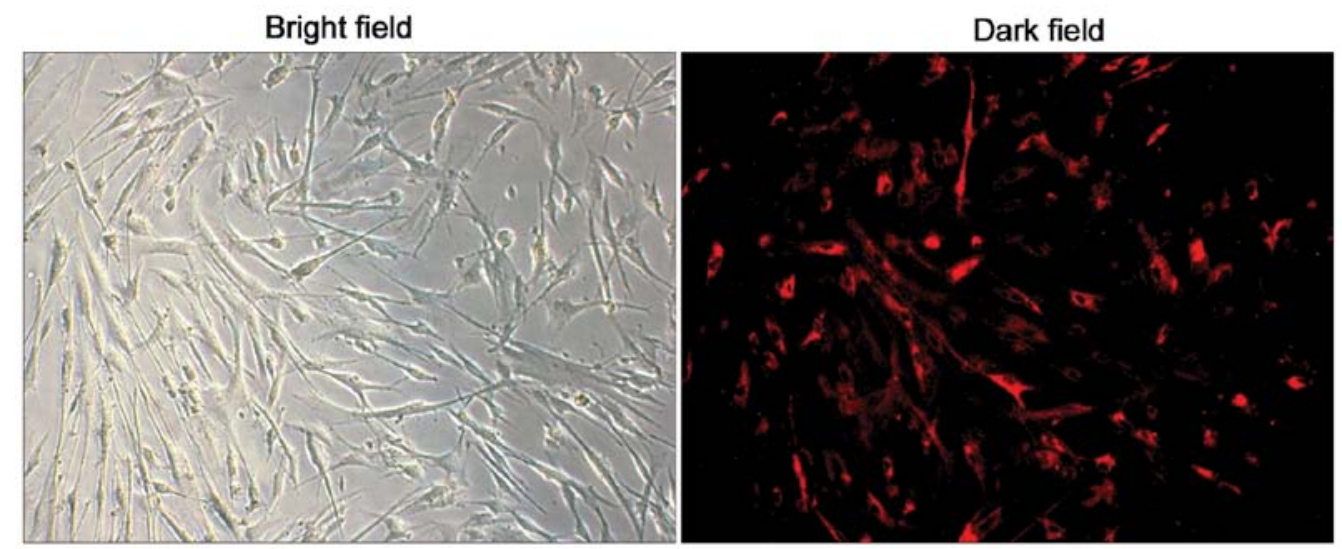

Figure 2. VSMCs $\left(2 \times 10^{6}\right.$ cells $\left./ \mathrm{ml}\right)$ were electroporated with Cy3-labeled Ras-a1 using one square waveform pulse of $200 \mathrm{~V}$ electric fields and $20 \mathrm{msec}$ pulse lengths. The transfection efficiency of $(82.11 \pm 6.03 \%)$ was calculated as the ratio of electroporated cells in the dark field to the cells in the bright field.

Table I. Doses of aptamer pools and Ras proteins in each selection.

\begin{tabular}{lccc}
\hline Round & $\begin{array}{c}\text { ssDNA } \\
(\mathrm{pmol})\end{array}$ & $\begin{array}{c}\text { Ras protein } \\
(\mathrm{pmol})\end{array}$ & ssDNA/Ras \\
\hline 1 & 1,000 & 100 & 10 \\
2 & 500 & 50 & 10 \\
3 & 200 & 20 & 10 \\
4 & 100 & 10 & 10 \\
5 & 100 & 7.5 & 13 \\
6 & 50 & 3.75 & 13 \\
7 & 50 & 2.5 & 20 \\
8 & 30 & 1.5 & 20 \\
9 & 30 & 1.2 & 25 \\
10 & 20 & 0.8 & 25 \\
11 & 20 & 0.6 & 33 \\
\hline
\end{tabular}

ssDNA, single-stranded DNA.

Electrotransfer of Ras-al into VSMC. To observe the electrotransfer of Ras-a1 into VSMCs, Cy3-labeled Ras-a1 was visualized using a fluorescence microscope. The transfection efficiency was defined as the ratio of electroporated cells labeled with $\mathrm{Cy} 3$ red fluorescence in the dark field to the cells in the bright field (Fig. 2). The transfection efficiency was $(82.11 \pm 6.03 \%)$ with a survival rate of $(83.04 \pm 1.74 \%)$.

Ras-al inhibits VSMC proliferation. The proliferation of VSMCs was significantly inhibited in cells transfected with Ras-a1 compared with that of control. The inhibition rates obtained at $12,24,36$ and $48 \mathrm{~h}$ were $25,40,29$ and $23 \%$, respectively (Fig. 3B). The maximal inhibitory rate was achieved at $24 \mathrm{~h}$, and prolonged treatment resulted in no greater growth inhibition (Fig. 3).

Ras-al inhibits VSMC migration. Cell migration of VSMCs was significantly inhibited in Ras-a1 cells in a time-dependent manner. Compared with the control group, no statistical difference was noted in the number of migrated cells in the
Ras-a1-transfected group at $6 \mathrm{~h}(65.86 \pm 21.75$ vs. $68.73 \pm 17.8$, $\mathrm{P}>0.05)$. However, compared with the control group, statistical differences were noted in the cellular migration of the Ras-a1 transfected group at $12 \mathrm{~h}(97.53 \pm 21.89$ vs. $117.47 \pm 22.66$, $\mathrm{P}<0.05), 18 \mathrm{~h}(160.60 \pm 19.91$ vs. $226.80 \pm 29.29, \mathrm{P}<0.01)$, and $24 \mathrm{~h}$ (228.73 \pm 28.31 vs. $329.53 \pm 63.73, \mathrm{P}<0.01$ ), respectively (Fig. 4).

Effects of Ras-al on MEK1/2, ERK1/2 and Akt phosphorylation in VSMC. To investigate the effect of Ras-al on the MAPK and PI3K signaling pathways, we measured the phosphorylation of MEK1/2, ERK1/2 and Akt proteins using western blot analysis. As shown in Fig. 5, a significant decrease was detected in the phosphorylation of MEK1/2, ERK1/2 and Akt proteins in Ras-a1-transfected cells compared with the control $(\mathrm{P}<0.05)$. By contrast, the expression of Ras protein was not altered by Ras-a1 transfection. These data suggested that Ras-a1 inhibited the proliferation and migration of VSMCs by modulating the signal transduction of MAPK and PI3K pathways.

\section{Discussion}

Restenosis, defined as the reoccurrence of the luminal narrowing of a vessel, remains a challenge to the long-term success of therapeutic approaches to CHD such as PCI and CABG. It has been reported that VSMC proliferation and migration was proportional to the extent of vascular injury (14). Therefore, we aimed to develop a potential therapeutical strategy for neointimal hyperplasia and subsequent restenosis by inhibiting the proliferation and migration of VSMCs.

Extensive studies have been performed to investigate the potential application of aptamer in disease diagnosis and treatment. Aptamers with high affinity and specificity have been developed to target peptides, proteins, drugs, organic and inorganic molecules and even whole cells (7-9). Accumulating evidence has indicated that certain aptamers showed great therapeutic efficacy both in vitro and in vivo. Pegaptanib, the first therapeutic aptamer approved by the FDA for the treatment of age-related macular degeneration, acts by inhibiting VEGF165 $(15,16)$. This aptamer is primarily responsible for pathological ocular neovascularization and increasing vascular permeability. OPN-R3, an RNA aptamer against human 
Table II. The OD and Kd values of different aptamers.

\begin{tabular}{lccccccccc}
\hline Aptamer & 1st P & 4th P & 6th P & 8th P & 9th P & 10th P & 11th P & Ras-a1 & Ras-a27 \\
\hline OD & 0.220 & 0.498 & 0.702 & 0.917 & 1.012 & 0.988 & 1.080 & 1.213 \\
Kd (nM) & 51.5 & 47.4 & 39.9 & 24.8 & 19.8 & 19.6 & 18.3 & 15.3 & 48.3 \\
\hline
\end{tabular}

P, pool; OD, optical density.
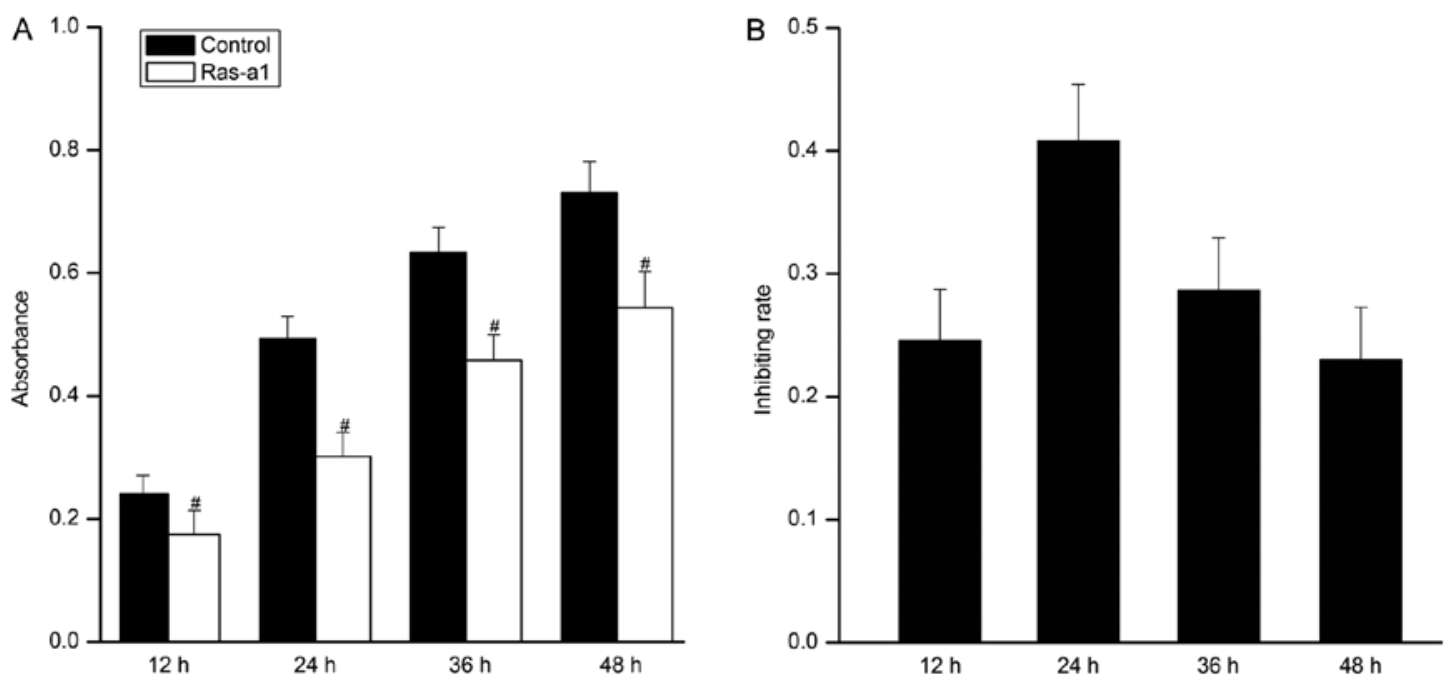

Figure 3. Effects of Ras-a1 on VSMCs proliferation measured by CCK-8 assay. (A) Compared with the control, a significant decrease was noted in the proliferation of VSMCs in cells transfected with Ras-a1 at 12,24, 36 and $48 \mathrm{~h}$, respectively, according to the absorbance obtained at a $450 \mathrm{~nm}$ wavelength (P<0.01). (B) The maximal inhibitory rate was obtained at $\sim 40 \%$ at $24 \mathrm{~h}$. Data are presented as means \pm standard deviation. ${ }^{*} \mathrm{P}<0.01 \mathrm{vs}$. control.

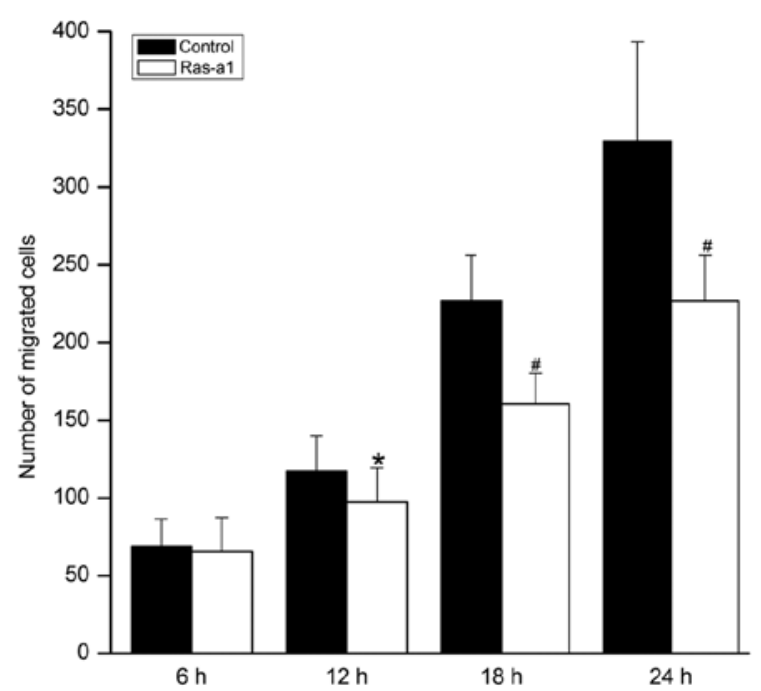

Figure 4. Effect of Ras-a1 on VSMCs migration determined using a Transwell assay. Ras-a1 and control cells were electroporated using one square waveform pulse of $200 \mathrm{~V}$ and $20 \mathrm{msec}$. The DAPI-stained cells were counted under a converted fluorescence microscope at a magnification of $x 200$. Compared with the control, the migration of VSMCs transfected with Ras-a1 was significantly inhibited in a time-dependent manner compared with the control. Data are presented as means \pm standard deviation. ${ }^{*} \mathrm{P}<0.05$ vs. control; ${ }^{\#} \mathrm{P}<0.01$ vs. control.

osteopontin, has been reported to significantly decrease local progression and distant metastases (17).
Few studies have been performed regarding the interruption or inhibition of the proliferation and migration of VSMCs by interrupting the Ras signals using aptamers. Sedding et al (18) demonstrated that 3-deazaadenosine (c3Ado) may prevent VSMC proliferation and neointima formation by interrupting Ras signaling. In addition, using recombinant adenoviruses containing a constitutively active mutant (AdRasV12) to infect rat common carotid arteries following balloon injury, Jin et al (19) showed that the neointimal formation was significantly elevated compared with the negative control at week 2 . In the present study, we initially isolated ssDNA aptamers that bound specifically to Ras proteins using SELEX. During the in vitro selection, blank control groups were set in order to eliminate false positives. Different pools of aptamers were obtained and the highest binding affinity was identified in the 11th pool. A total of 45 individual aptamers were thus obtained, among which Ras-al had the highest affinity with a Kd of $15.3 \mathrm{nM}$. The biological activity of Ras protein was silenced by ssDNA aptamer Ras-a1, and the proliferation and migration of VMSCs was significantly reduced.

To investigate the mechanism of Ras-a1-mediated inhibition of VSMC proliferation and migration, we examined the effects of Ras-a1 on the expression and activation of Ras, MEK1/2, ERK1/2 and Akt, respectively. It has been well established that ERK1/2 and PI3K/Akt signaling pathways play key roles in the regulation of VSMC proliferation and migration (20,21). According to previous findings, the activation of Ras induced by growth factors through direct 
Table III. The results of cloning and sequencing.

Aptamer Random sequence $\left(5^{\prime} \rightarrow 3^{\prime}\right)$

Family 1

1 TCGTCGGATCGACGCGGTTTAGTGAGTGTGCGTGG

2 TCGTCGGATCGACGCGGTTTAGTGAGTGTGCGTGG

3 GCTGAAGCAGGGTTGAGTGATAAATGGTGCGTCGG

4 GGGCAAGCGTCGTGGACAGGAAGAAGTATGTGGTA

5 GTAAGTGTGTGGTGATTTGGTATATGTAGGCGTCG

Family 2

6 GGGGGGGTCGGGAGGTCAGGTTAGGGGGGGTGTTG

7 GAGGGGCGGAGTATCGGGGGGGGGGGAGGGGGCGG

8 GAAAGAAGGGGTTGTTGTGAGGGGGGTGGTGGTGG

9 GCAAAGATTGGGGGGATGATTGAAATAAGCGTGTG

10 GCGGGGGTGGTTGGGCTGGGGGATGTGGGGTGGTG

11 TGGGGACGGGGGTATGGGAGGGGGGTGGTGGTGGA

12 GCTAATGCCGTATGCGCAAGGATGACACACGGGGG

13 AAGAGTGAGATGATGGATAATGCAAACTGGGGGTG

14 AGTGCTGCTTGGGGGTTCGTAGTTCGAGTGCTAGG

15 CGATGGTGTGGTTGGTGGGGGAGTGCGACGCCTGA

16 TAAGCGCGAGGgGGTATAGGTGTATGTAGCGTGAG

Family 3

17 GAgGgatagGaGTGGTGTTGTTCGTTGTTGCATGG

18 GTGGAGTATTGGTAGTGATAGAGGGCTTAGGCGGG

19 GGAGGGGATGACGAGAGCAATCGGCGTGCAAGGGA

20 GGGAGTTGGCGAATAAGCTTTAGTTGGGAGGGATG

21 TGGTTGAGGGGAGGCAGAGGGAGGTATAAGGTATG

22 ACCGGGCGGGTTGCAAGATGGTGAGGGTGCGGGTG

23 GGAGGGGAAGAAGACGCGAACATGAAAGATGCGTG

Family 4

24 GAGACAGAGCTACGGAGGTGAGATATCAGCATGTG

25 TTGAGCGGGTAATTGGAGTATGTGTGTGTAGGTGG

26 TACTGCAAGAGTGAAAATGTGGTTAGTGAGGTCGG

27 GATGATGTGGGGAGTGGAGTGTAATGGAGAGTGAG

28 GGCGGATGTGAGTTGTTATGAGTTGCAGAGTGGAG

29 GGAGTGTGTTGGGGTGCTATTTGGTTGTATGTGTG

Family 5

30 GGTGAGGAGAAGCGGTAGGTGAAATGGAAGTAAGG

31 AGCAAAGGAAGACAGGGGAAGTGGTGAAGTTGACG

32 AGAGGATAGTGATGGACGGTGAAGTGCGTGGGTGG

33 AGGGGAAGTGGTGATCGGATAGGACAAAGGAAGTG

Family 6

34 GAGGTATGCACGATGGGAGTGAAACTAACGGGGAG

35 TAAGGGAAAGTGTAGTAAGGAAAGTTGAGTGGGAG

36 CGTGGGAGCTGGAACATATTGCTGAGGAAGTTCGT

Family 7

37 GCCTCGTGGCGATAAAGGTTGGGTAGGTTGGGTCC

38 GAGGTTGGGCTGGAATAAGATTGGTTGTGGGTAGG

39 TGCGAGAAGCGCGGAAAAGGGTAGGCACGGAAGCG

Family 8

40 GCGTGGTAAGGTCGGCAGCAGGGCGCAGTTGGAGG

41 TGCCGGAACCGGCAGGGAAGGTGTGTGGGTGTGCA

42 GCGGAGGAAGGTAGAGACGATGGCTATAGCGATAG

Family 9

43 GTCACATCGAACAACGGGCGTAATTCGTGTCTGGG

44 GAAAGACGGAGGCGTGTAGGAAAGTGGAGTGAGCG

Family 10

45 TCTTTCTATTTTCTGTTGGTTTTTGTTGTGTGTTT

Letters in bold indicate conservative sequences.
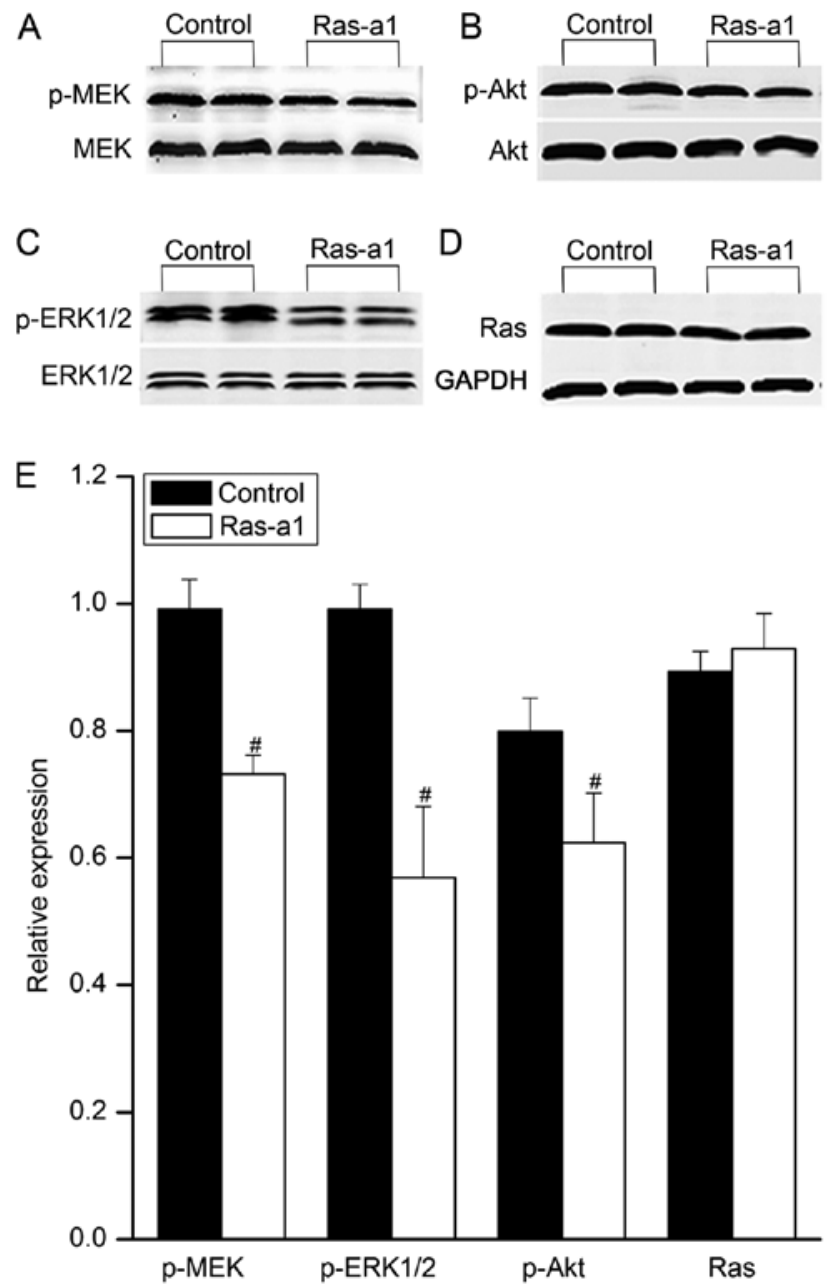

Figure 5. Effects of Ras-a1 on the phosphorylation of MEK1/2, ERK1/2 and Akt protein in VSMCs measured using western blot analysis. The expression of (A) phosphorylated MEK1/2, (B) ERK1/2 and (C) Akt, as well as (D) total Ras protein was assessed. The expression was normalized using GAPDH. Data are presented as means \pm standard deviation. ${ }^{\#} \mathrm{P}<0.01$ vs. control.

binding with the receptors contributed to the activation of the downstream components of MEK and PI3K, which activate ERK1/2 and Akt, respectively (22). As a result, various downstream effectors controlling the proliferation and migration of VSMCs were triggered. Our results revealed that the phosphorylation of Ras, MEK1/2, ERK1/2 and Akt showed a significant decrease in Ras-a1-transfected VSMCs within 24 h. Consequently, we hypothesized that Ras-a1 inhibits the phosphorylation and activation of Ras, resulting in the subsequent blocked activation of the downstream signaling proteins in the Ras-MEK-ERK1/2 and Ras-PI3K-Akt pathways. Therefore, the migration and proliferation of VSMCs was blocked due to the transduction failure of extracellular signals into the nucleus.

In conclusion, a novel Ras ssDNA aptamer designated as Ras-a1 with high specificity and affinity to Ras protein was isolated using SELEX. It inhibited the proliferation and migration of VSMCs by downregulating the phosphorylation of Ras protein and interrupting the signal transduction in Ras-MEK1/2-ERK1/2 and PI3K/Akt pathways. The results demonstrate Ras-a1 may be used as an effective strategy to prevent restenosis after PCI and CABG. 


\section{Acknowledgements}

This study was supported by grants from the National Natural Science Foundation of China (no. 30872537) and the Fundamental Research Funds for the Central Universities (no. 2042014kf0117).

\section{References}

1. Shahabuddin S, Sami SA, Ansari JA, et al: Coronary artery bypass grafting after percutaneous coronary intervention. J Coll Physicians Surg Pak 22: 340-341, 2012.

2. Abbate A, Biondi-Zoccai GG, Agostoni P, Lipinski MJ and Vetrovec GW: Recurrent angina after coronary revascularization: a clinical challenge. Eur Heart J 28: 1057-1065, 2007.

3. Wang Z, Zhang X, Chen S, et al: Lithium chloride inhibits vascular smooth muscle cell proliferation and migration and alleviates injury-induced neointimal hyperplasia via induction of PGC-1 $\alpha$. PLoS One 8: 55471-55482, 2013.

4. Louis SF and Zahradka P: Vascular smooth muscle cell motility: From migration to invasion. Exp Clin Cardiol 15: e75-e85, 2010.

5. Guo X, Chen KH, Guo Y, Liao H, Tang J and Xiao RP: Mitofusin 2 triggers vascular smooth muscle cell apoptosis via mitochondrial death pathway. Circ Res 101: 1113-1122, 2007.

6. Ramos KS: H-RAS controls phenotypic profiles of vascular smooth muscle cells and the pathogenesis of vascular proliferative disorders. Circ Res 104: 1139-1141, 2009.

7. Kong HY and Byun J: Nucleic acid aptamers: new methods for selection, stabilization, and application in biomedical science. Biomol Ther (Seoul) 21: 423-434, 2013.

8. Chen $\mathrm{CH}$, Chernis GA, Hoang VQ and Landgraf R: Inhibition of heregulin signaling by an aptamer that preferentially binds to the oligomeric form of human epidermal growth factor receptor-3. Proc Natl Acad Sci USA 100: 9226-9231, 2003.

9. Cerchia L, Duconge F, Pestourie C, et al: Neutralizing aptamers from whole-cell SELEX inhibit the RET receptor tyrosine kinase. PLoS Biol 3: e123, 2005.

10. Bell C, Lynam E, Landfair DJ, Janjic $\mathrm{N}$ and Wiles ME: Oligonucleotide NX1838 inhibits VEGF165-mediated cellular responses in vitro. In Vitro Cell Dev Biol Anim 35: 533-542, 1999.
11. Lee PP, Ramanathan M, Hunt CA and Garovoy MR: An oligonucleotide blocks interferon-gamma signal transduction. Transplantation 62: 1297-1301, 1996.

12. Torres RA, Drake DA, Solodushko V, et al: Slingshot isoform-specific regulation of cofilin-mediated vascular smooth muscle cell migration and neointima formation. Arterioscler Thromb Vasc Biol 31: 2424-2431, 2011.

13. Izumiya Y, Kim S, Izumi Y, et al: Apoptosis signal-regulating kinase 1 plays a pivotal role in angiotensin II-induced cardiac hypertrophy and remodeling. Circ Res 93: 874-883, 2003.

14. Indolfi C, Esposito G, Di Lorenzo E, et al: Smooth muscle cell proliferation is proportional to the degree of balloon injury in a rat model of angioplasty. Circulation 92: 1230-1235, 1995.

15. Deissler HL and Lang GE: Effect of VEGF165 and the VEGF aptamer pegaptanib (Macugen) on the protein composition of tight junctions in microvascular endothelial cells of the retina. Klin Monbl Augenheilkd 225: 863-867, 2008 (In German).

16. Apte RS: Pegaptanib sodium for the treatment of age-related macular degeneration. Expert Opin Pharmacother 9: 499-508, 2008.

17. Mi Z, Guo H, Russell MB, Liu Y, Sullenger BA and Kuo PC: RNA aptamer blockade of osteopontin inhibits growth and metastasis of MDA-MB231 breast cancer cells. Mol Ther 17: 153-161, 2009. Sedding DG, Tröbs M, Reich F, et al: 3-Deazaadenosine prevents smooth muscle cell proliferation and neointima formation by interfering with Ras signaling. Circ Res 104: 1192-1200, 2009.

18. Sedding DG, Tröbs M, Reich F, Walker G, Fink L, Haberbosch W, Rau W, Tillmanns H, Preissner KT, Bohle RM, et al: 3-Deazaadenosine prevents smooth muscle cell proliferation and neointima formation by interfering with Ras signaling. Circ Res 104: 1192-1200, 2009.

19. Jin G, Chieh-Hsi Wu J, Li YS, Hu YL, Shyy JY and Chien S: Effects of active and negative mutants of Ras on rat arterial neointima formation. J Surg Res 94: 124-132, 2000.

20. Isenović ER, Kedees MH, Tepavcevic S, et al: Role of PI3K/AKT, cPLA2 and ERK1/2 signaling pathways in insulin regulation of vascular smooth muscle cells proliferation. Cardiovasc Hematol Disord Drug Targets 9: 172-180, 2009.

21. Shen YJ, Zhu XX, Yang X, et al: Cardamonin inhibits angiotensin II-induced vascular smooth muscle cell proliferation and migration by downregulating p38 MAPK, Akt, and ERK phosphorylation. J Nat Med 3: 623-629, 2014.

22. Shah BH, Neithardt A, Chu DB, Shah FB and Catt KJ: Role of EGF receptor transactivation in phosphoinositide 3-kinase-dependent activation of MAP kinase by GPCRs. J Cell Physiol 206: 47-57, 2006. 